\title{
Mechanisms of Motion-Defined Shape Perception Elucidated by the Regression Analyses between Psychophysical and Psychophysiological Data
}

\author{
Shin'ichiro Kamiya*, Toshiyo Tamura and Takao Sato ${ }^{1}$ \\ Department of Medical System Engineering, Graduate School of Engineering, Chiba University \\ 'Department of Psychology, Graduate School of Humanities and Societies, University of Tokyo
}

(Received September 29, 2011; accepted March 19, 2012)

Key words: motion-defined shape perception, visual field, random dot kinematogram, evoked potentials, regression analysis

To elucidate the mechanisms of motion-defined shape perception (SP), we carried out experiments by employing the random-dot kinematogram. Participants were asked 1) whether a coherently moving dot in a core rectangle was moving "toward the upper left or lower right" (direction discrimination task) and 2) whether the shape of a motion-defined rectangular area composed of the moving dots was "oblong vertically or horizontally" (pattern discrimination task) with various moving velocities from 14.4 to $126 \mathrm{deg} / \mathrm{s}$. When stimuli were presented in the upper or lower half of the visual field (VF), the rate of correct responses for six participants were significantly higher in the lower VF than in the upper VF for the pattern discrimination task (lower VF enhancement) at 25 and $40 \mathrm{deg} / \mathrm{s}(p<0.05)$ but not for the direction discrimination task. When the visualevoked potential was measured in the psychophysiological experiments with similar stimuli, the amplitudes of the peak potential at $290 \mathrm{~ms}$ were significantly correlated with the data of correct-response rates for the pattern discrimination task $(p<0.05)$ alone. It was suggested that the information was initially treated for motion itself in the magnocellular system and then returned presumably to the parvocellular system in the primary visual cortex to accomplish the motion-defined SP at approximately $290 \mathrm{~ms}$.

\section{Introduction}

Motion-defined shape perception (SP) is realized when we watch an area composed of coherently moving dots surrounded by a "sandstorm" region of incoherently moving dots. Obviously, SP is an essential element in visual information processing, and is indispensable for pattern recognition.

${ }^{*}$ Corresponding author: e-mail: skamiya@graduate.chiba-u.jp 
Several types of SP have been identified on the basis of their specific visual dimensions. They are broadly classified as luminance-defined SP, ${ }^{(1-5)}$ motion-defined SP, ${ }^{(3,6-8)}$ disparity-defined SP, ${ }^{(9,10)}$ texture-defined $\mathrm{SP}^{(11,12)}$ orientation- and color-defined SP, ${ }^{(10)}$ and illusory contour perception. ${ }^{(13)}$ In general, these types of SP are processed by one or both of the two neuronal systems, the parvocellular system, which processes shape and color, and the magnocellular system, which deals with motion and location..$^{(14,15)}$

Luminance-defined SP is the most fundamental among them. In psychophysiological studies of this SP, Jeffreys ${ }^{(1)}$ demonstrated that the peak components of the visual-evoked potential (VEP) revealed the polarity reversal corresponding to upper and lower visual field (VF) presentations. This polarity reversal was regarded as reflecting the retinotopy of the early visual cortex..$^{(16)}$

Furthermore, in psychophysical experiments for this luminance-defined SP, McAnany and Levine ${ }^{(17)}$ reported that only under the parvocellular-dominant condition, did the responses in the experiments exhibit visual anisotropy, namely, enhanced sensitivity to stimuli presented in the lower VF (lower VF enhancement).

Motion-defined SP is somewhat complicated, as it includes two processes: motion detection and shape perception. It has been established that motion detection is performed in the magnocellular system represented by the middle temporal area $(\mathrm{MT})^{(18-24)}$ or V3A. ${ }^{(25-32)}$ Concerning the role of the parvocellular system in this SP, however, no direct evidence has been presented.

A variety of psychophysical and psychophysiological analyses have also been applied to the investigation of motion-defined SP. In their psychophysical experiments, Chang and Julesz ${ }^{(6)}$ employed an apparent motion stimulus made using a random-dot kinematogram (RDK) of two frames and examined two tasks: the pattern of the area composed of coherently moving dots and the direction of their motion. When the amount of displacement was increased, the rates of correct responses to both tasks decreased. However, the threshold of the displacement (Dmax) was much larger for the direction discrimination task than for the pattern discrimination task. In their psychophysiological study, Niedeggen and Wist ${ }^{(33)}$ found that the onset of coherent motion per se elicited a negative peak component at around $200 \mathrm{~ms}$ in VEP. By contrast, Bach and Meigen ${ }^{(34)}$ emphasized that the VEPs induced by four types of SP (namely, luminance-defined, orientation-defined, motion-defined, and disparity-defined) shared a common peak component appearing at $230-310 \mathrm{~ms}$. However, the results obtained in psychophysical experiments have remained isolated from the above psychophysiological findings, and no attempt has been made to clarify the relationship between them. To gain more insight into motion-defined SP, it is important to perform both types of experiment with the same stimuli on the same participants and to carry out regression analyses between the psychophysical and psychophysiological data obtained.

In this study, we provided a series of motion-defined stimuli by employing RDK and carried out the psychophysical experiments for two tasks of direction discrimination and pattern discrimination as well as the psychophysiological experiment for VEP measurement around the occipital area induced by the same stimuli. Regression analyses were performed between the data on the rate of correct responses for each task (acquired in the psychophysical experiments) and the component of VEPs (revealed in the psychophysiological experiment). The results of the analyses were mainly 
utilized to identify (1) the time course and (2) the activated areas of the motion-defined SP. In addition, we tried to examine the interaction between the magnocellular and parvocellular systems in this SP. Finally, the general implication of such regression analyses in perception studies is discussed.

\section{Materials and Methods}

\subsection{Participants}

Six students of the same university with normal or corrected-to-normal vision (visual acuity $>1.0$ ) participated in the experiments. All of them (four males and two females, average age 21.4 years old) gave their written informed consent to participate in the experiments in advance. Participants were all right-handed.

\subsection{Stimuli}

The visual stimuli were generated with a Power Macintosh G4 computer using the Psychophysicstoolbox program library, ${ }^{(35)}$ which, together with Videotoolbox, enabled the interruption of scanning lines via "VBL task". Stimuli were presented on a CRT screen (EIZO, FlexScan T565) at a frame rate of $60 \mathrm{~Hz}$, resolution of $640 \times 480$ pixels, and viewing distance of $57 \mathrm{~cm}$. The stimuli were composed of RDKs. The size of the random dots was 3.6 arc $\mathrm{min}$; half were white $\left(22.14 \mathrm{~cd} / \mathrm{m}^{2}\right)$ and half were black $(0 \mathrm{~cd} /$ $\left.\mathrm{m}^{2}\right)$ (Fig. 1). The RDK window $(240 \times 240$ pixels) was placed in the upper half or lower half of the center of the screen, surrounded by an averaged gray background $(11.07 \mathrm{~cd} /$ $\left.\mathrm{m}^{2}\right)$. In the center of the RDK window, we positioned the core rectangular region (120 $\times 60$ pixels), which was elongated either vertically or horizontally. In the core region presented at 7.2 deg eccentricity, the dots moved coherently in either an upward and left or a downward and right direction. The velocities of the moving dots varied across six levels ranging from 14.4 to $126 \mathrm{deg} / \mathrm{s}(14.4,18,25.2,39.6,68.4$, and $126 \mathrm{deg} / \mathrm{s})$. The rest of the RDK region was a "sandstorm" area composed of incoherently moving dots. The duration of stimulation ("ON" period) was $500 \mathrm{~ms}$, and each presentation was followed by an "OFF" period, during which time the dots in the entire RDK region moved incoherently for $500 \mathrm{~ms}$. The fixation point, set at the center of the screen as a red point $(2 \times 2$ pixels), was continuously turned on during both "ON" and "OFF" periods.

\subsection{Procedure of psychophysical experiments}

The experiments focused on two independent tasks: (1) pattern discrimination and (2) direction discrimination. The pattern discrimination task consisted of 12 conditions (six moving-dot velocities and two core patterns). The participants were asked to answer whether the contour of the core rectangular region was oblong vertically or horizontally. In this task, the moving direction was fixed to the lower right [Fig. 1(a)].

The direction discrimination task also included 12 conditions (six moving-dot velocities and two moving directions). The participants were asked to answer whether the direction of the moving dots was toward the upper left or lower right. In this task, the core region was fixed at a horizontally elongated pattern [Fig. 1(b)].

For both tasks, each condition set was repeated 20 times, giving a total of 240 trials 

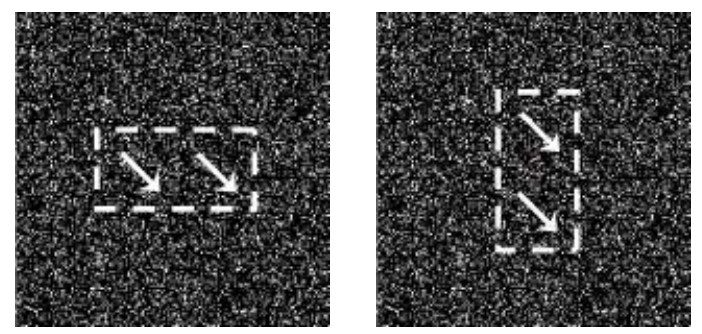

(a)
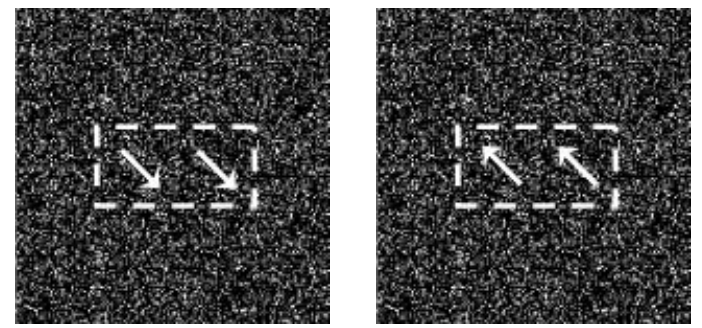

(b)

Fig. 1. Schematic drawings of RDK stimuli used in psychophysical experiments for patterndiscrimination task (a) and direction discrimination task (b). The core rectangular region composed of coherently moving dots in the direction indicated by arrows is surrounded by the "sandstorm" zone composed of incoherently moving dots. For the pattern discrimination task in panel (a), the participants were asked whether the pattern of the core rectangular region was oblong vertically or horizontally, whereas for the direction discrimination task in panel (b), they were asked whether the direction of moving dots was toward the lower right or upper left. The velocity of the moving dots was varied in 6 levels $(14.4,18,25.2,39.6,68.4$, and $126 \mathrm{deg} / \mathrm{s})$. The stimulus used in the case of the right side of panel B was also used in the psychophysiological experiments.

presented randomly in a series (constant stimuli method). When one trial was initiated, the response was entered through the keyboard of the PC, using the two-alternative forced-choice method during either the "ON" or "OFF" period. Then the next trial was followed immediately. The first sequence of 240 trials was performed with either upper or lower VF presentation, and the VF presentation was reversed for the second sequence. The participants were instructed to give their responses as accurately as possible.

\subsection{Procedure of psychophysiological experiments}

In the psychophysiological experiments, the velocity of the RDK stimuli was also varied across six levels, but the core rectangular shape and motion direction were fixed to horizontal and lower right, respectively, because preliminary experiments had shown that no difference in VEPs was elicited by using two shapes or two directions. Each 
participant was exposed to the same RDK stimuli 100 times to record one VEP in either the upper or lower VF. The active electrode was placed on $\mathrm{Oz}$ (the $10 \%$ system), above the early visual cortex, and the reference electrode was placed on the left earlobe. ${ }^{(1)}$ The impedance of each electrode was confirmed to be less than $5 \mathrm{k} \Omega$ at $10 \mathrm{~Hz}$. EEG was recorded with Biotop 6R12-2 amplifiers (NEC Corporation, Japan). Data were digitized with a sampling rate of $1,000 \mathrm{~Hz}$ and stored on a PC. A high-cut filter was used for the measurement to diminish the high-frequency noise over $30 \mathrm{~Hz}$. Trials containing eyeblinks were excluded off-line. In our preliminary experiment, we confirmed that the time difference between the trigger signal for the recording system and that for the presentation was less than $5 \mathrm{~ms}$.

\section{Results}

\subsection{Results of psychophysical experiments}

Figure 2 shows the relationship between the moving-dot velocity (abscissa in logarithmic scale) and the correct-response rate (ordinate) obtained in the psychophysical experiments. The correct-response rate data shown are averages of the six participants. Panel A shows the results of the pattern discrimination task in the upper and lower VF presentations. Panel B shows the results of the direction discrimination task in each of the two VFs. The data in each graph were fitted to a sigmoid function curve, and the threshold (75\% correct-response rate) was estimated from each curve. The threshold in the pattern discrimination task in Panel A was $23.8 \mathrm{deg} / \mathrm{s}$ in the upper VF and $34.4 \mathrm{deg} / \mathrm{s}$

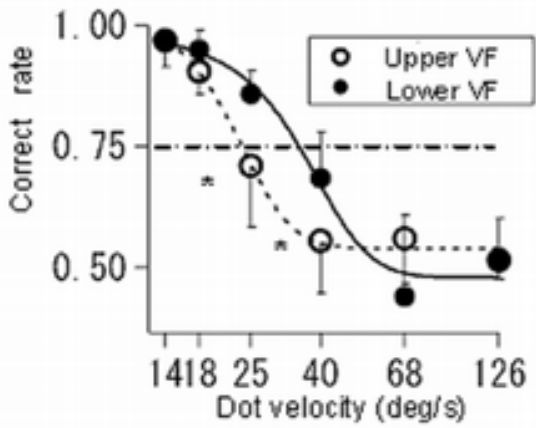

(a)

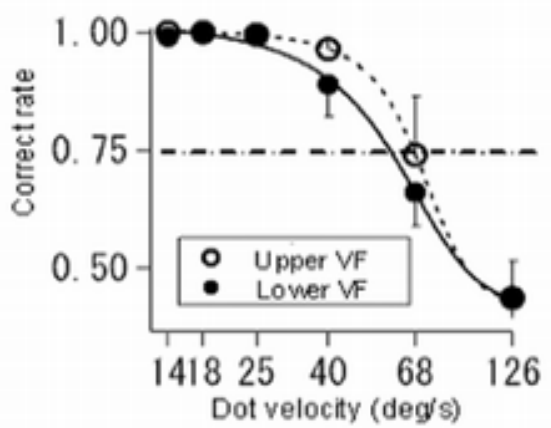

(b)

Fig. 2. Results of psychophysical experiments for pattern-discrimination task (a) and directiondiscrimination task (b). The correct rate is plotted vs the dot velocity in a logarithmic scale. The dotted line with blank circles (O) indicates the case of the upper-VF presentation, and the solid line with solid circles $(\bullet)$ shows the case of the lower VF. The significant difference between the upper and lower presentations is indicated by asterisks $(*: p<0.05)$. The horizontal line designates the threshold level of the correct rate of 0.75 . 
in the lower VF. The threshold in the direction discrimination task in Panel B was 67.7 $\mathrm{deg} / \mathrm{s}$ in the upper VF and $58.1 \mathrm{deg} / \mathrm{s}$ in the lower VF. These results suggested that the direction discrimination task was much easier than the pattern discrimination task in both VF presentations.

In Fig. 2(a), the effect of VF on the correct-response rate was examined by statistically analyzing (two-tailed paired t-test) the data of the six participants for each moving velocity. The results revealed that the correct-response rate for the pattern discrimination was significantly higher in the lower VF presentation than in the upper $\mathrm{VF}$ presentation at the velocities of $18 \mathrm{deg} / \mathrm{s}[t(5)=2.37, p<0.05], 25.2 \mathrm{deg} / \mathrm{s}[t(5)=3.31$, $p<0.05]$, and $39.6 \mathrm{deg} / \mathrm{s}[t(5)=2.81, p<0.05]$. Figure $2(\mathrm{~b})$, however, shows no significant difference in the correct-response rate for direction discrimination at any velocity. The results of these psychophysical experiments demonstrated that lower VF enhancement was observed for pattern discrimination but not for direction discrimination.

\subsection{Results of psychophysiological experiments}

Panels A-F in Fig. 3 depict the grand means of VEPs induced by RDK stimuli for the six participants at each velocity $(14.4,18.0,25.2,39.6,68.4$, and $126.0 \mathrm{deg} / \mathrm{s})$. The solid lines represent VEPs elicited by lower VF presentation, and the dotted lines represent VEPs elicited by upper VF presentation. It should be noted that components later than $300 \mathrm{~ms}$ involve event-related potentials, ${ }^{(36)}$ which are irrelevant to VEPs.

Within the time range of $300 \mathrm{~ms}$, the lower VF responses, represented by solid lines, revealed clear negative peaks within the range of 260-290 ms, although they became less conspicuous as the velocity increased. As for the upper VF responses, represented by dotted lines, the positive peaks were also observed at around $290 \mathrm{~ms}$, except in the case shown in panel D (39.6 deg/s). The reversed polarities of the peak components elicited by the upper and lower VF presentation in this study were consistent with the results of previous VEP studies obtained by luminance-defined checkerboard stimulation. ${ }^{(1,2)}$

Changes in the peak amplitudes of the VEPs in relation to moving-dot velocity are shown in Fig. 4. The solid circles represent the absolute values of the negative peaks elicited by lower VF presentation, and the empty circles indicate the peak values induced by upper VF presentation. The dot velocities are plotted in a logarithmic scale. In the velocity range from 14.4 to $39.6 \mathrm{deg} / \mathrm{s}$, the peak amplitudes of the VEPs elicited by lower VF stimuli were much larger than those elicited by upper VF stimuli. The ratio of the lower/upper amplitudes was maximally 5.8 at $25.2 \mathrm{deg} / \mathrm{s}$.

\subsection{Correlation between psychophysical and psychophysiological data}

To investigate the relationships between the results of the psychophysical and psychophysiological experiments, we carried out regression analyses of the data on the rates of correct responses with the absolute amplitudes of the VEP peak around $290 \mathrm{~ms}$. As shown in Fig. 5(a), the correct-response rate data for the pattern discrimination task and the absolute peak amplitudes showed a significant correlation in both the lower VF $[r=0.89, t(4)=3.88, p<0.01]$ and upper $\mathrm{VF}[r=0.73, t(4)=2.15, p<0.05]$. By contrast, no statistically significant correlation was found for the direction discrimination task (panel B) for either the lower or upper VF presentation $[r=0.67, t(4)=1.81$, n.s.; $r=0.53, t(4)=1.25$, n.s., 

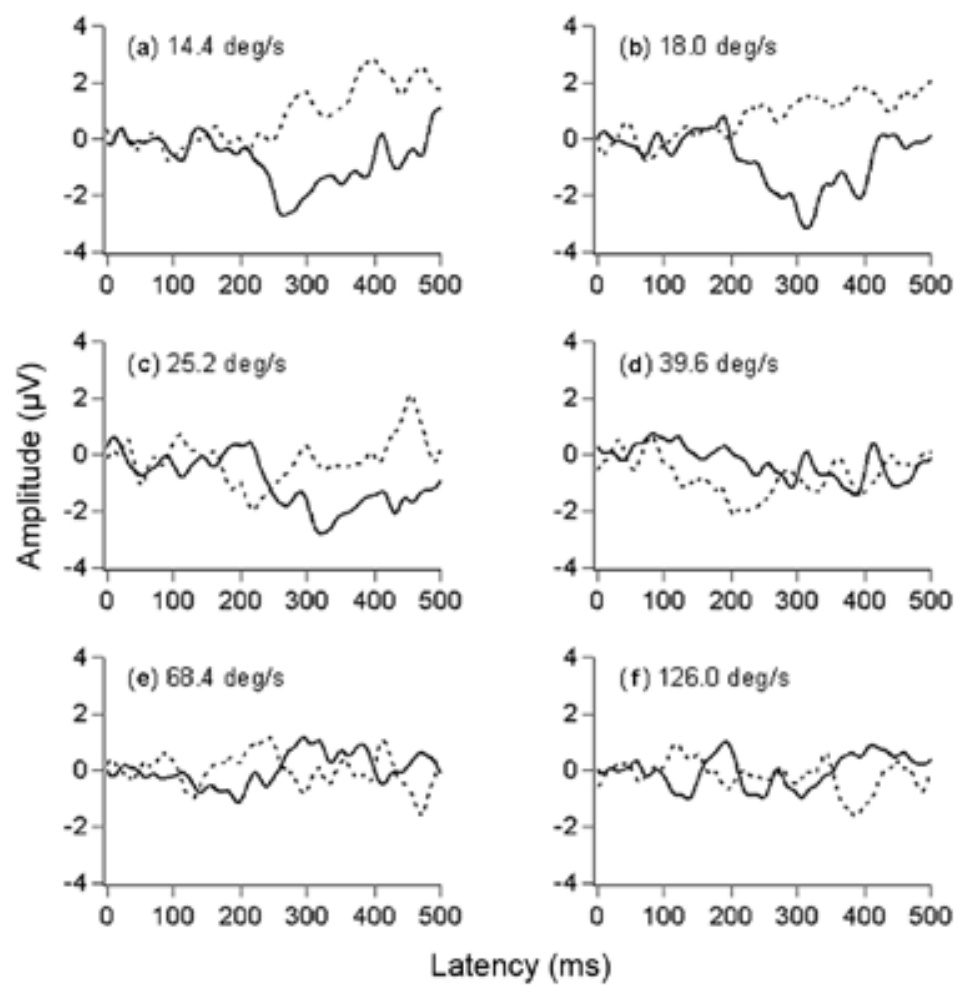

Fig. 3. VEPs elicited by RDK stimuli at six moving-dot velocities. All the solid lines represent the grand average of VEPs for the six participants evoked by the lower-VF presentation, and the dotted lines show those evoked by the upper-VF presentation. The number in each panel indicates the moving-dot velocity. In all the panels, the ordinate is the peak amplitude in $\mu \mathrm{V}$ and the abscissa indicates the elapsed time in $\mathrm{ms}$.

respectively]. These results suggest that the peak deflection of the VEP at around $290 \mathrm{~ms}$ was definitely linked to the information processing of motion-defined SP.

\section{Discussion}

\subsection{Time course of motion-defined SP}

The results in Fig. 5(a) demonstrated that the peak amplitudes of VEPs around 290 $\mathrm{ms}$ in the present psychophysiological experiments were significantly correlated with the correct-response rates for the pattern-discrimination task alone. This result indicated that the latency of this shape perception was approximately $290 \mathrm{~ms}$. On the other hand, 


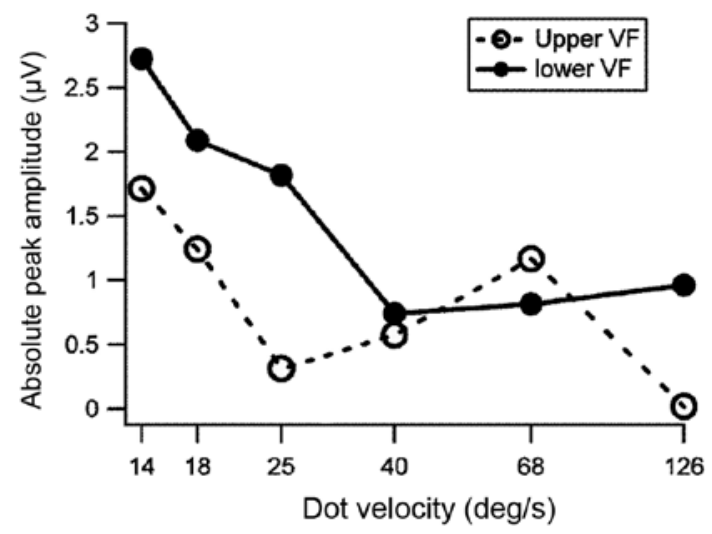

Fig. 4. Changes in absolute amplitudes of VEP peaks at around $290 \mathrm{~ms}$ corresponding to movingdot velocity. The solid points connected with solid lines represent the absolute amplitudes of the grand average curve for the lower-VF presentation, and the open circles with dotted lines represent those for the upper VF.

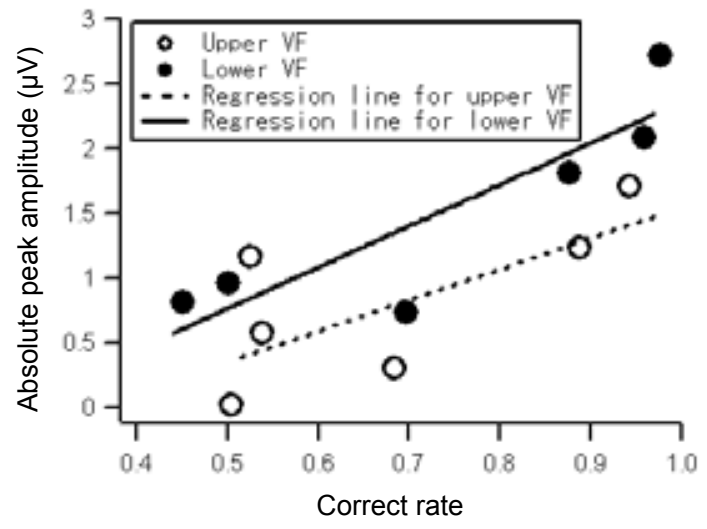

(a)

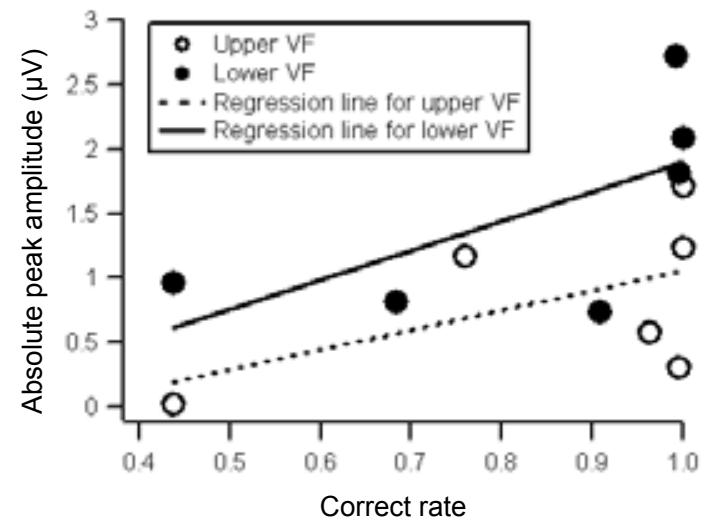

(b)

Fig. 5. Diagrams of correlation between correct rate and peak amplitude of VEP at around 290 ms. In panel (a), the absolute amplitude of the VEP peak at around $290 \mathrm{~ms}$ was significantly correlated with the rate of correct responses for the pattern discrimination task for both the upper- and lower-VF presentations. The regression line of the peak amplitude $(Y)$ against the correct rate $(X)$ was $Y=3.20 X-0.84$ in the lower-VF presentation $(r=0.89, p<0.01)$ and $Y=2.41 X-0.86$ in the upper-VF presentation $(r=0.73, p<0.05)$. The correlations between the same parameters for the direction discrimination task, shown in panel (b), were not significant in either the lowerVF $(r=0.67$, n.s. $)$ or the upper-VF $(r=0.53$, n.s. $)$ presentation. 
it has been explored in many psychophysical studies that the information processing of coherent motion onset is achieved at about $200 \mathrm{~ms} .^{(33,37-41)}$ The difference in latency from 200 to $290 \mathrm{~ms}$ may be regarded as the time lag for information transition from the site for motion detection to that for pattern perception. To confirm this transition model more precisely, however, it is necessary to carry out experiments with larger numbers of participants.

Bach and Meigen ${ }^{(34)}$ reported, in their psychophysiological study, that a common component was found in the VEPs elicited by 4 differently defined stimuli (luminance-, orientation-, motion-, or disparity-defined). When the effects of the onset and offset on VEPs ("low-level" VEP) were cautiously cancelled out, the induced VEPs ("texturesegregation" VEP) all revealed similar waveforms with peaks in the range from 230 to $310 \mathrm{~ms}$. These extensive and important findings, however, must be confirmed in regard to reliability by using other findings acquired from studies of entirely different approaches. In this aspect, the results in this study can be good counterpart evidence, at least for motion-defined SP, because the latency (approximately $290 \mathrm{~ms}$ ) of our VEPs, of which the specific linkage to SP was certified by the regression analyses between psychophysical and psychophysiological data, was highly consistent with that of VEP determined independently of such regression analyses. This agreement suggests that similar correlation studies should be applied to other types of SP to elucidate the psychophysical implication of induced VEPs. In our experiments, the luminance and other static elements of the visual field were kept unchanged at the onset and offset of the stimulation. This maneuver was probably effective in minimizing the induction of "low-level" VEPs. Since the term "texture segregation" is nearly identical to our "SP", it is not surprising that their findings were highly consistent with the results of the present study.

\subsection{Elicited areas in process of motion-defined SP}

In this study, the VEP waveforms at around $290 \mathrm{~ms}$ in Fig. 3 exhibited polarity reversal, which has been regarded as proof of information processing in the early visual cortex, including V1, V2, and V3. (1,16) $^{(1)}$

Several areas in the early visual cortex have been reported to be concerned with pattern perception in motion-defined SP. In human imaging experiments, the kinetic occipital (KO) area located between MT and the V3 complex was found to be activated by a motion-defined shape rather than MT. ${ }^{(42-45)}$ Furthermore, the lateral occipital complex (LOC) area covering the extrastriate visual cortex was also activated in motion-defined SP as well as luminance-defined SP.(46) In electrophysiological studies in Macaque monkeys, the V3 complex (V3, VP) area has also been suggested to be an important area for the integration and transformation of visual signals for motion and pattern perception. ${ }^{(47,48)}$

In a clinical study in patients with parieto-occipital cortex lesions including Brodmann's areas 18 and 19, Regan et al. ${ }^{(49)}$ reported that several patients, who were intact in motion detection itself, were not able to discriminate motion-defined letter shapes. Consequently, it was concluded that the area for motion detection must be clearly isolated from the area for motion-defined shape perception, and must be involved 
in the injured regions of the patients. Note that areas 18 and 19 are largely overlapped with such areas as V3 complex, KO, and LOC mentioned above. These findings then well reinforce our idea that the motion-defined SP is accomplished in the early visual cortex after being treated in the magnocellular system (MT or V3A) for the information of motion itself.

\subsection{Roles of magnocellular and parvocellular systems}

This is the first report demonstrating the lower-VF enhancement in motion-defined SP. The results of our psychophysical experiments in Fig. 2(a) revealed that the correctresponse rate for the pattern discrimination task was significantly higher for the lowerVF presentation than for the upper-VF presentation at two velocities (25.2 and 39.6 $\mathrm{deg} / \mathrm{s}$ ), whereas no significant differences in the correct-response rates were found for the direction discrimination task [Fig. 2(b)]. This result suggests that the discrimination processes for these two tasks are differently treated in information processing.

It is conceivable that the VEP amplitude difference between the upper- and lowervisual field (VF) presentations is induced by the different locations of activated areas for the individual presentations. However, according to Portin et al., ${ }^{(50)}$ although the depths of dipoles for lower left and upper right stimuli were about equal, the equivalent current dipole amplitudes were stronger for the lower left than the upper right stimuli, making it very improbable that their results were due to differences in source depths.

It has been established that direction discrimination is processed in the magnocellular system in $\mathrm{MT}^{(51,52)}$ or V3A. ${ }^{(25,27)}$ In contrast, various studies have suggested that the participation of the parvocellular system in image processing is reflected as lower VF enhancement. For instance, it has been reported in the micro-electrode study ${ }^{(15)}$ that the parvocellular system plays dominant roles in the treatment of high-spatial-frequency images. In addition, Skrandies ${ }^{(53)}$ and Carrasco et al. ${ }^{(54)}$ have also demonstrated that the increased spatial frequency in the sine wave grating makes the enhancement more prominent. By combing these two kinds of findings, the lower-VF enhancement may be assumed to be a sign representing the parvocellular system participation. McAnany and Levine ${ }^{(17)}$ have shown that the lower-VF enhancement takes place only under parvocellular-dominant conditions in luminance-defined SP. It is then likely that even in motion-defined SP, the parvocellular system is incorporated in its shape perception process. To examine such a model, however, we require further detailed spatial information about excited areas associated with reliable temporal resolution of latencies.

\subsection{Significance of regression analyses between psychophysical and psychophysiological data}

One of the noteworthy findings in this study is, as shown in Fig. 5, a close correlation between the data on the rate of correct responses for pattern discrimination and the peak amplitude of VEP at around $290 \mathrm{~ms}$. This means that the more accurate the perception, the more prominent the peak. In other words, the magnitude of the VEP peak reflects the accuracy of motion-defined SP. The question then arises whether such a quantitative relationship between the psychological reaction and the physiological response is confined to motion-defined SP or can be extended to other types of visual SP or different 
types of perception by any other sensory modality. To the best of our knowledge, this type of regression analysis between psychophysical and psychophysiological data has not been reported in conventional perception studies.

The time course of the perception processes in motion-defined SP was identified with the aid of regression analyses, as shown in Fig. 5(a). A similar approach would be possible for other types of perception with complex processes, as long as a significant correlation is established between the correct-response rate for a psychophysical task, which explicitly represents a process, and a VEP component with a stable latency. The regression study proposed here may be regarded as a new approach for the time course analyses of perception. If such temporal information is combined with spatial information of excited areas by virtue of noninvasive measurement methods with higher spatial resolutions (e.g., fMRI, PET etc.), this will obviously lead to a better understanding of perception mechanisms.

\section{References}

1 D. Jeffreys: Visual Evoked Potentials in Man: New Development, ed. J. E. Desmedt (Oxford Univ. Press, Oxford, 1977) p. 134.

2 D. Jeffreys: Evoked Potentials, ed. C. Barber (MIT Press, Cambridge, 1980) p. 149.

3 D. Regan: Vision Res. 29 (1989) 1389.

4 W. Schneider, D. Noll and J. Cohen: Nature 365 (1993) 150.

5 C. Aine, S. Supek and J. George: Int'1 J. Neurosci. 80 (1995) 79.

6 J. Chang and B. Julesz: Vision Res. 23 (1983) 639.

7 V. Lamme, B. Van Dijk and H. Sperkreijse: Nature 363 (1993) 541.

8 V. Marcar, D. Xiao, S. Raiguel, H. Maes and G. Orban: J. Neurophysiol. 74 (1995) 1258.

9 B. Julesz: Bell System Tech. J. 39 (1960) 1125.

10 K. Zipser, V. Lamme and P. Schiller: J. Neurosci. 16 (1996) 7376.

11 B. Julesz: Sci. Am. 212 (1965) 38.

12 B. Julesz: Sci. Am. 232 (1975) 34.

13 G. Kanizsa: Rivista di Psicologia 49 (1955) 7.

14 M. Livingstone and D. Hubel: J. Neurosci. 7 (1987) 3416.

15 M. Livingstone and D. Hubel: Science 240 (1988) 740.

16 J. Ales, J. Yates and A. Norcia: Neuroimage 52 (2010) 1401.

17 J. McAnany and M. Levine: Vision Res. 47 (2007) 2327.

18 S. Zeki: Brain Res. 14 (1969) 271.

19 S. Zeki: J. Physiol. 236 (1974) 549.

20 R. Gattass and C. Gross: J. Neurophysiol. 46 (1981) 621.

21 J. Allman, F. Miezin and E. McGuinness: Annu. Rev. Neurosci. 8 (1985) 407.

22 W. Newsome and E. Paré: J. Neurosci. 8 (1988) 2201.

23 M. Fiorani, R. Gattass, M. Rosa and A. Sousa: J. Comp. Neurol. 287 (1989) 98.

24 C. Salzman, K. Britten and W. Newsome: Nature 346 (1990) 174.

25 R. Tootell, J. Mendola, N. Hadjikhani, P. Ledden, A. Liu, J. Reppas, M. Sereno and A. Dale: J. Neurosci. 17 (1997) 7060.

26 S. Ahlfors, G. Simpson, A. Dale, J. Belliveau, A. Liu, A. Korvenoja, J. Virtanen, M. Huotilainen, R. Tootell, H. Aronen and R. Ilmoniemi: J. Neurophysiol. 82 (1999) 2545.

27 J. Braddick, J. O’Brien, J. Wattam-Bell, J. Atkinson, T. Hartley and R. Turner: Perception 30 (2001) 61. 
28 D. Chawla, C. Buechel, R. Edwards, A. Howseman, O. Josephs, J. Ashburner and K. Friston: Neuroimage 9 (1999) 508.

29 L. Cornette, P. Dupont, A. Rosier, S. Sunaert, P. Van Hecke, J. Michiels, L. Mortelmans and G. Orban: J. Neurophysiol. 79 (1998) 2749.

30 R. Goebel, D. Khorram-Sefat, L. Muckli, H. Hacker and W. Singer: Eur. J. Neurosci. 10 (1998) 1563.

31 G. Rees, K. Friston and C. Koch: Nat. Neurosci. 3 (2000) 716.

32 S. Sunaert, P. Van Hecke, G. Marchal and G. Orban: Exp Brain Res. 127 (1999) 355.

33 M. Niedeggen and E. Wist: Cog. Brain Res. 8 (1999) 95.

34 M. Bach and T. Meigen: Vision Res. 37 (1997) 1409.

35 D. Pelli: Spatial Vision 10 (1997) 437.

36 S. Sutton, M. Braren, J. Zubin and E. John: Science 150 (1965) 1187.

37 T. Probst, H. Plendl, W. Paulus, E. Wist and M. Scherg: Exp. Brain Res. 93 (1993) 345.

38 Z. Kubová, M. Kuba, H. Spekreijse and C. Blakemore: Vision Res. 35 (1995) 197.

39 M. Niedeggen and E. Wist: Neurosci. Lett. 246 (1998) 61.

40 M. Hoffmann, T. Dorn and M. Bach: Vision Res. 39 (1999) 437.

41 J. Lorteije, J. Kenemans, T. Jellema, R. van der Lubbe, F. de Heer and R. van Wezel: J. Cog. Neurosci. 18 (2006) 158.

42 G. Orban, P. Dupont, B. De Bruyn, R. Vogels, R. Vandenberghe and L. Mortelmans: Proc. Natl. Acad. Sci. USA. 92 (1995) 993.

43 P. Dupont, B. De Bruyn, R. Vandenberghe, A. Rosier, J. Michiels, G. Marchal, L. Mortelmans and G Orban: Cereb. Cortex 7 (1997) 283.

44 S. Van Oostende, S. Sunaert, P. Van Hecke, G. Marchal and G. Orban: Cereb. Cortex 7 (1997) 690.

45 S. Zeki, R. Perry and A. Bartels: Cereb. Cortex 13 (2003) 189.

46 K. Grill-Spector, T. Kushnir, S. Edelman, Y. Itzchak and R. Malach: Neuron 21 (1998) 191.

47 D. Felleman and D. Van Essen: J. Neurophysiol. 57 (1987) 889.

48 K. Gegenfurtner, D. Kiper and J. Levitt: J. Neurophysiol. 77 (1997) 1906.

49 D. Regan, D. Giaschi, J. Sharpe and X. Hong: J. Neurosci. 12 (1992) 2198.

50 K. Portin, S. Vanni, V. Virsu and R. Hari: Exp. Brain Res. 124 (1999) 287.

51 J. Maunsell and W. Newsome: Annu. Rev. Neurosci. 10 (1987) 363.

52 P. Schiller, N. Logothetis and E. Charles: Vis. Neurosci. 5 (1990) 321.

53 W. Skrandies: Progress in Sensory Physiology, ed. D. Ottoson (Springer-Verlag, Berlin, 1987) p. 1.

54 M. Carrasco, C. Talgar and E. Cameron: Spatial Vision 15 (2001) 61. 